\title{
Frequency and versatility of letters in the English language
}

\author{
ROBERT L. SOLSO \\ University of Idaho, Moscow, Idaho 83849 \\ and \\ JOSEPH F. KING \\ Loyola University of Chicago, Chicago, Illinois 60626
}

\begin{abstract}
Tabulations of letter and letter-combination versatility and frequency were made based on the Kucera and Francis (1967) word frequency count. Letter versatility, a new descriptive statistic, was defined as the number of different words in which a letter appears. These tabulations may be useful in the investigation of visual information processing, reading skills, and human memory.
\end{abstract}

A powerful instrument in the understanding of verbal processing has been the statistical analysis of the language. These descriptive statistics can be traced to the 14th century, when Simonetta (as reported by Cherry, 1966) published letter frequencies that were used in cryptoanalysis, Since then, language has been statistically analyzed in terms of bigram and trigram frequency, vowel and dipthong frequency, syllabic frequency, and other variables (see Cherry, 1966; Kahn, 1967; Miller, 1951; Underwood \& Schulz, 1960, for more complete historical reviews).

A significant methodological change from counting raw frequencies of letters was introduced by Mayzner and Tresselt (1965); single-letter frequencies were measured by the position each letter occupied in words of varying length. Positional frequency has been hypothesized by Mason (1975) to augment visual feature information in the identification of letters. Spatial redundancy in her study was defined as "the degree of correlation present in printed English between visual features (fixed levels on physical dimension) and their spatial location within multiletter configuration." By having sixth grade readers search through single six-letter strings for either the presence or absence of a specific letter target, she demonstrated that spatial redundancy rather than familiarity is the relevant redundancy variable. However, the concept of versatility was not introduced.

In a recent publication (Topper, Macey, \& Solso,

A portion of this paper was presented at the annual meeting of the Psychonomic Society, Denver, November 1975. The authors thank Lin Morris for her suggestions on the manuscript and composition of the figure. Reprint requests should be sent to Robert L. Solso, Department of Psychology, University of Idaho, Moscow, Idaho 83843.
1973), bigram frequency and a new descriptive statistic, bigram versatility, were reported. Bigram versatility is defined as the number of different words in which a given bigram appears. Bigram frequency, on the other hand, is the total number of occurrences of a bigram in the language sample. The potency of bigram versatility as it relates to memory search time has been demonstrated by Solso, Topper, and Macey (1974).

In the present paper, a definitive count of singleletter frequency and versatility is reported. This information may be of primary interest to those researchers investigating visual information processing, reading skills, memory search, verbal learning, and related areas.

\section{METHOD}

All frequencr and versatility counts are based on the Kucerra and Francis (1967) rrequency count of about one-million words in the English language. All hyphenated words, words containing apostrophies. and numbers were excluded from the analysis. Approximately 40,000 different words with an approximate total frequency of $1,000,000$ occurrences were used.

The procedure for counting total frequency was as follows: The word NOTE has a frequency of 127 per million. The frequency totals for the letters " $N$ " " $O$ " " $T$ " " $E$ ", are incremented by 127 . The word ATTACK has a frequency of 105 per million: " $A$ " is incremented by 210 as is " $T$," while " $C$ " and " $K$ " are increased by 105 . Since versatility represents the number of different words in which a particular letter combination occurs, the versatility of " $A$, ," "T, " " $C$," and " $K$ " increase by one.

The normative data in this report are confined to total frequency and versatility for all words in the Kucera and Francis count and to letter and frequency counts by position for four- and fiveletter words. In Table 1 , the total single-letter frequencies and versatilities are represented, with percentage of usage based on total usage in parentheses. The ratio between frequency and versatility is also shown in Table 1 (FV ratio), as are the FV ratios based on percentage usage in parentheses.

Tables 2 and 3 contain frequency counts by position for four- 
Table 1

Single-Letter Frequency and Versatility (and Percentage) Occurrence in the English Language

\begin{tabular}{|c|c|c|c|}
\hline Letter & Frequency & Versatility & FV Ratio \\
\hline A & $348411(7.61)$ & $20372(7.80)$ & $17.10(.97)$ \\
\hline B & $70714(1.54)$ & $5478(2.09)$ & $12.91(.74)$ \\
\hline $\mathrm{C}$ & $142336(3.11)$ & $11279(4.32)$ & $12.62(.72)$ \\
\hline D & $181054(3.95)$ & $11140(4.26)$ & $16.32(.93)$ \\
\hline $\mathrm{E}$ & $577583(12.62)$ & $25794(9.88)$ & $22.39(1.28)$ \\
\hline $\mathrm{F}$ & $107219(2.34)$ & $3830(1.46)$ & $27.99(1.60)$ \\
\hline G & $89499(1.95)$ & $8043(3.08)$ & $11.13(.63)$ \\
\hline $\mathbf{H}$ & $252191(5.51)$ & $7017(2.68)$ & $35.94(2.05)$ \\
\hline I & $336166(7.34)$ & $20587(7.88)$ & $16.33(.93)$ \\
\hline $\mathrm{J}$ & $7296(.15)$ & $649(.24)$ & $11.24(.63)$ \\
\hline K & $29838(.65)$ & $2942(1.12)$ & $10.14(.58)$ \\
\hline $\mathrm{L}$ & $188261(4.11)$ & $14259(5.46)$ & $13.20(.75)$ \\
\hline $\mathbf{M}$ & $116574(2.54)$ & $7975(3.05)$ & $14.62(.83)$ \\
\hline $\mathrm{N}$ & $325652(7.11)$ & $18768(7.19)$ & $17.35(.99)$ \\
\hline 0 & $350121(7.65)$ & $16050(6.14)$ & $20.29(1.25)$ \\
\hline $\mathbf{P}$ & $93040(2.03)$ & $7756(2.97)$ & $12.00(.68)$ \\
\hline $\mathrm{Q}$ & $4936(.10)$ & $545(.20)$ & $9.06(.50)$ \\
\hline $\mathrm{R}$ & $281881(6.15)$ & $19243(7.37)$ & $14.65(.83)$ \\
\hline$S$ & $297531(6.50)$ & $18977(7.27)$ & $15.68(.89)$ \\
\hline $\mathrm{T}$ & $427179(9.33)$ & $17257(6.61)$ & $24.76(1.41)$ \\
\hline $\mathrm{U}$ & $124736(2.72)$ & $9665(3.70)$ & $12.91(.73)$ \\
\hline V & 45707 (.99) & $3423(1.31)$ & $13.35(.75)$ \\
\hline W & $86563(1.89)$ & $2769(1.06)$ & $31.26(1.78)$ \\
\hline $\mathrm{X}$ & 9032 ( .19) & $847(.32)$ & $10.66(.59)$ \\
\hline $\mathrm{Y}$ & $78749(1.72)$ & $5344(2.04)$ & $14.74(.84)$ \\
\hline $\mathrm{Z}$ & $4316(.09)$ & $973(.37)$ & $4.44(.24)$ \\
\hline
\end{tabular}

Table 2

Letter Frequency and Versatility by Position for Four-Letter Words

\begin{tabular}{|c|c|c|c|c|c|c|c|c|c|c|}
\hline \multirow[b]{3}{*}{ Letter } & \multicolumn{8}{|c|}{ Position } & & \\
\hline & \multicolumn{2}{|c|}{1} & \multicolumn{2}{|c|}{2} & \multicolumn{2}{|c|}{3} & \multicolumn{2}{|c|}{4} & \multicolumn{2}{|c|}{ Totals } \\
\hline & $\mathrm{F}$ & V & $\mathrm{F}$ & V & $F$ & V & $F$ & $\mathrm{~V}$ & $F$ & V \\
\hline A & 3210 & 108 & 27208 & 468 & 20437 & 205 & 1141 & 98 & 51996 & 879 \\
\hline B & 7428 & 175 & 255 & 11 & 319 & 42 & 343 & 35 & 8345 & 263 \\
\hline $\mathrm{C}$ & 4726 & 146 & 100 & 12 & 6748 & 87 & 102 & 17 & 11676 & 262 \\
\hline D & 4720 & 132 & 408 & 19 & 2567 & 78 & 10212 & 142 & 17907 & 371 \\
\hline $\mathrm{E}$ & 3774 & 65 & 21088 & 307 & 22051 & 204 & 33108 & 347 & 80021 & 923 \\
\hline $\mathrm{F}$ & 10660 & 119 & 4 & 3 & 1671 & 29 & 563 & 27 & 12898 & 178 \\
\hline $\mathrm{G}$ & 3243 & 101 & 136 & 10 & 1236 & 59 & 1497 & 57 & 6112 & 227 \\
\hline $\mathrm{H}$ & 9913 & 128 & 30082 & 88 & 420 & 19 & 12353 & 66 & 52768 & 301 \\
\hline I & 2218 & 36 & 22376 & 302 & 9800 & 162 & 94 & 45 & 34488 & 545 \\
\hline $\mathrm{J}$ & 2153 & 56 & 2 & 1 & 12 & 4 & 1 & 1 & 2168 & 62 \\
\hline $\mathrm{K}$ & 2327 & 60 & 93 & 11 & 3096 & 37 & 5658 & 126 & 11174 & 234 \\
\hline L & 7988 & 139 & 2973 & 111 & 11378 & 191 & 7456 & 124 & 29795 & 565 \\
\hline$\overline{\mathrm{M}}$ & 10932 & 130 & 72 & 19 & 6969 & 81 & 8437 & 72 & 26410 & 302 \\
\hline $\mathrm{N}$ & 2185 & 60 & 5785 & 45 & 8459 & 231 & 14394 & 156 & 30823 & 492 \\
\hline 0 & 4313 & 63 & 24409 & 420 & 10904 & 146 & 3294 & 74 & 42920 & 703 \\
\hline $\mathrm{P}$ & 3625 & 142 & 981 & 16 & 1086 & 55 & 1779 & 82 & 7471 & 295 \\
\hline$Q$ & 20 & 5 & 1 & 1 & 0 & 0 & 4 & 2 & 25 & 8 \\
\hline $\mathrm{R}$ & 3192 & 115 & 6703 & 125 & 13867 & 209 & 5415 & 83 & 29177 & 532 \\
\hline$S$ & 10963 & 203 & 757 & 15 & 10248 & 146 & 11847 & 346 & 33815 & 710 \\
\hline$T$ & 30738 & 141 & 578 & 24 & 12350 & 122 & 25084 & 218 & 68750 & 505 \\
\hline $\mathrm{U}$ & 1349 & 15 & 7307 & 234 & 2840 & 78 & 75 & 26 & 11571 & 353 \\
\hline V & 1357 & 43 & 2848 & 12 & 5754 & 40 & 11 & 5 & 9970 & 100 \\
\hline W & 22441 & 95 & 575 & 24 & 1567 & 40 & 2173 & 31 & 26756 & 190 \\
\hline $\mathrm{X}$ & 1 & 1 & 70 & 7 & 479 & 10 & 100 & 17 & 650 & 35 \\
\hline $\mathrm{Y}$ & 2028 & 27 & 766 & 29 & 1010 & 25 & 10295 & 104 & 14099 & 185 \\
\hline \multirow[t]{2}{*}{ Z } & 77 & 12 & 4 & 3 & 313 & 17 & 145 & 16 & 539 & 48 \\
\hline & 155581 & 2317 & 155581 & 2317 & 155581 & 2317 & 155581 & 2317 & 622324 & \\
\hline
\end{tabular}


Table 3

Letter Frequency and Versatility by Position For Five-Letter Words

\begin{tabular}{|c|c|c|c|c|c|c|c|c|c|c|c|c|}
\hline \multirow[b]{3}{*}{ Letter } & \multicolumn{10}{|c|}{ Position } & & \\
\hline & \multicolumn{2}{|c|}{1} & \multicolumn{2}{|c|}{2} & \multicolumn{2}{|c|}{3} & \multicolumn{2}{|c|}{4} & \multicolumn{2}{|c|}{5} & \multicolumn{2}{|c|}{ Totals } \\
\hline & $F$ & $\mathrm{~V}$ & $\mathrm{~F}$ & $\mathrm{~V}$ & $F$ & V & $\mathbf{F}$ & V & F & V & $F$ & $\mathrm{~V}$ \\
\hline$A$ & 7698 & 239 & 11407 & 725 & 12769 & 451 & 5830 & 267 & 904 & 178 & 38608 & 1860 \\
\hline B & 5586 & 325 & 2166 & 16 & 947 & 96 & 524 & 61 & 49 & 13 & 9272 & 511 \\
\hline $\mathrm{C}$ & 6733 & 327 & 582 & 45 & 1052 & 116 & 8548 & 186 & 624 & 40 & 17539 & 714 \\
\hline D & 2604 & 210 & 676 & 44 & 2800 & 114 & 3147 & 165 & 12022 & 253 & 21249 & 786 \\
\hline $\mathrm{E}$ & 2290 & 104 & 10330 & 501 & 15037 & 285 & 19236 & 764 & 21890 & 603 & 68783 & 2257 \\
\hline $\bar{F}$ & 5856 & 222 & 1531 & 11 & 368 & 41 & 497 & 45 & 527 & 32 & 8779 & 351 \\
\hline $\mathrm{G}$ & 3824 & 175 & 714 & 14 & 3953 & 119 & 1578 & 115 & 3391 & 48 & 13460 & 471 \\
\hline $\mathbf{H}$ & 3592 & 175 & 18960 & 189 & 1783 & 33 & 2541 & 48 & 7236 & 160 & 34112 & 605 \\
\hline I & 979 & 57 & 10353 & 424 & 13954 & 380 & 7200 & 271 & 172 & 55 & 32658 & 1187 \\
\hline $\mathrm{J}$ & 626 & 66 & 2 & 2 & 299 & 8 & 6 & 3 & 0 & 0 & 933 & 79 \\
\hline K & 761 & 82 & 106 & 16 & 1220 & 46 & 1397 & 135 & 2283 & 108 & 5767 & 387 \\
\hline $\mathbf{L}$ & 4471 & 207 & 5021 & 274 & 3175 & 297 & 12004 & 282 & 5122 & 168 & 29793 & 1228 \\
\hline $\mathbf{M}$ & 4513 & 225 & 1410 & 38 & 2303 & 140 & 1818 & 121 & 488 & 55 & 10532 & 579 \\
\hline $\mathrm{N}$ & 2415 & 88 & 3093 & 109 & 4764 & 308 & 8837 & 295 & 5687 & 269 & 24796 & 1069 \\
\hline 0 & 3171 & 75 & 19107 & 625 & 10986 & 329 & 2952 & 212 & 661 & 115 & 36877 & 1356 \\
\hline $\mathrm{P}$ & 4687 & 242 & 1329 & 63 & 1143 & 77 & 989 & 105 & 792 & 52 & 8940 & 539 \\
\hline $\mathrm{Q}$ & 559 & 20 & 118 & 6 & 4 & 3 & 2 & 1 & 0 & 0 & 643 & 30 \\
\hline $\mathbf{R}$ & 3219 & 176 & 8173 & 368 & 8999 & 374 & 9474 & 231 & 12029 & 244 & 41894 & 1393 \\
\hline$S$ & 11986 & 507 & 995 & 40 & 2290 & 176 & 8270 & 199 & 15947 & 936 & 39488 & 1858 \\
\hline $\mathrm{T}$ & 14220 & 245 & 5851 & 101 & 4968 & 192 & 6444 & 305 & 11311 & 267 & 42794 & 1110 \\
\hline $\mathrm{U}$ & 2103 & 42 & 3415 & 319 & 9489 & 211 & 3283 & 113 & 18 & 9 & 18308 & 694 \\
\hline V & 1033 & 75 & 697 & 17 & 3087 & 96 & 1305 & 53 & 10 & 2 & 6132 & 243 \\
\hline W & 12761 & 141 & 716 & 49 & 805 & 56 & 1144 & 42 & 408 & 17 & 15834 & 305 \\
\hline $\mathrm{X}$ & 11 & 4 & 163 & 13 & 406 & 32 & 10 & 3 & 176 & 16 & 766 & 68 \\
\hline $\mathrm{Y}$ & 1587 & 25 & 368 & 52 & 551 & 56 & 128 & 19 & 5523 & 410 & 8157 & 562 \\
\hline \multirow[t]{2}{*}{$\mathrm{Z}$} & 17 & 12 & 19 & 5 & 150 & 30 & 138 & 25 & 32 & 16 & 356 & 88 \\
\hline & 107302 & 4066 & 107302 & 4066 & 107303 & 4066 & 107302 & 4066 & 107302 & 4066 & 536510 & \\
\hline
\end{tabular}

and five-letter words. In Table 2, 2,317 four-letter words were found which tave a total frequency of $155,581 . F$ and $V$ positional percentages are obtained by dividing the specific letter/position by the sum of the column $F$ or V. Table 3 is based on 4,066 words with a total frequency of 107,302 .

Complete normative data on words of different length and larger letter combinations may be found in Solso (Note 1). Included are bigram frequencies and versatility, trigram frequencies and versatility, and positional norms.

The relationship between letter frequency and versatility is represented in Figure 1. In Figure 1, frequency by rank order is along the abscissa, while percentage occurrence of letters by versatility and frequency is represented as the ordinate. The general covariation of these two measures is apparent, as are anomalies (e.g., $\mathrm{H}$, which enjoys high frequency due to "the," "there," "this," etc., but relatively low versatility).

The frequency of encountering letters in written material is an overall measure of familiarity, but the versatility values reported here represent the frequency of familiarity of the context within which a letter appears in a word.

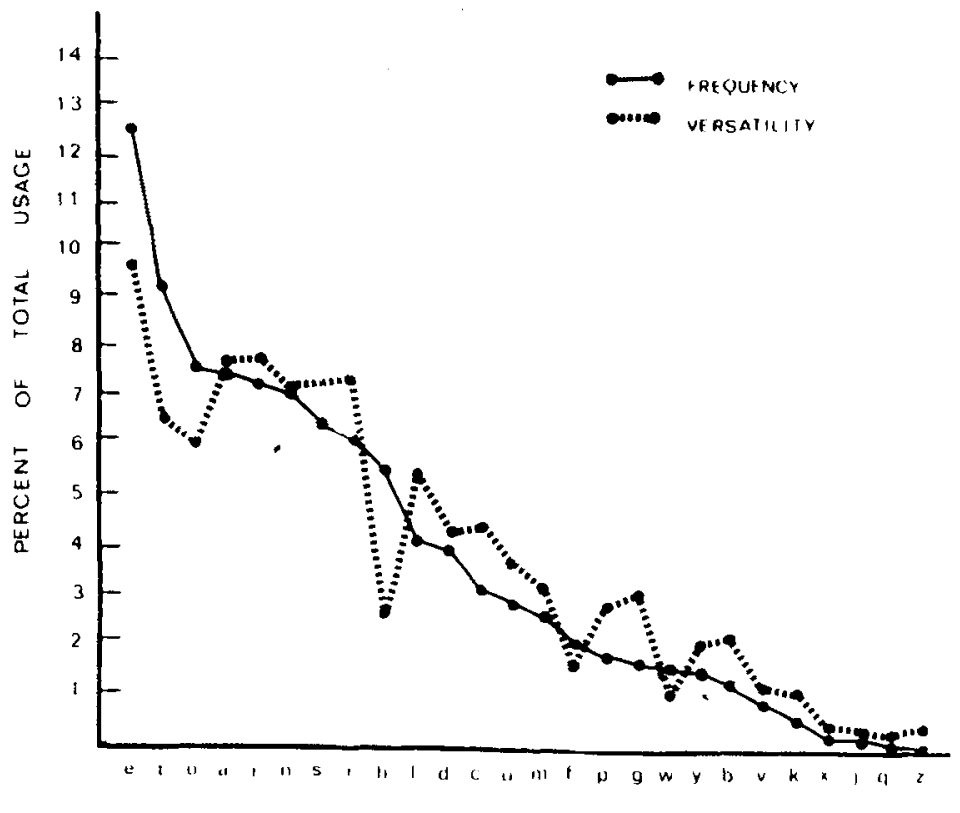

Figure 1. Total usage of English letters by rank order frequency and versatility. 


\section{REFERENCE NOTE}

1. Solso, R. L. Statistical analysis of letters and letter combinations in the English language. Unpublished manuscript.

\section{REFERENCES}

Cherry, C. On Human Communication. Cambridge: M.I.T. Press. 1970.

Kucera. H., \& Francis, W. N. Computational analysis of present-day American English. Providence: Brown University Press. 1967.

Mason. M. Reading ability and letter search time: Effects of orthographic structure defined by single-letter positional frequency. Joumal of Experimental Psychology: General, 1975, 1. 146-166.
Mayzner, M. S., \& Tresselt, M. E. Table of single-letter and bigram frequency counts for various word-length and letterposition combinations. Psychonomic Monograph Supplements, 1965, 1(Whole No. 2).

Miller, G. A. Language and communication. New York: McGraw-Hill, 1951.

Solso, R. L., Topper, G. E., \& Macey, W. H. Anagram solution as a function of bigram versatility. Joumal of Experimental Psychology, 1973, 100, 259-262.

Topper, G. E., Macey, W. H., \& Solso, R. L. Bigram versatility and bigram frequency. Behavior Research Methods \& Instrumentation, 1973, 5, 51-53.

Underwood, B. J., \& Schulz, R. W. Meaningfulness and verbal learning. Chicago: Lippencott, 1960.

(Received November 29, 1975; revision accepted February 10, 1976.) 